Mens

Revue d'histoire intellectuelle et culturelle

mens

\title{
Un homme et son journal : comment Jules-Paul Tardivel " domestiqua " La Vérité
}

\section{Dominique Marquis}

Volume 13, numéro 2, printemps 2013

URI : https://id.erudit.org/iderudit/1025982ar

DOI : https://doi.org/10.7202/1025982ar

Aller au sommaire du numéro

\section{Éditeur(s)}

Centre de recherche en civilisation canadienne-française

ISSN

1492-8647 (imprimé)

1927-9299 (numérique)

Découvrir la revue

Citer cet article

Marquis, D. (2013). Un homme et son journal : comment Jules-Paul Tardivel « domestiqua " La Vérité. Mens, 13(2), 35-57. https://doi.org/10.7202/1025982ar
Résumé de l'article

En 1881, Jules-Paul Tardivel lance à Québec La Vérité, un journal de combat ultramontain. Tardivel porte ce journal à bout de bras, mais il n'est pas seul : il est soutenu par un réseau ultramontain encore très actif à cette époque. En 1890, Tardivel fait face à des difficultés matérielles telles que la survie du journal est sérieusement mise en péril. Les amis ultramontains de Tardivel sont alors mis à contribution pour trouver une solution. Plusieurs propositions sont évoquées, mais Tardivel, ne voulant pas partager la direction du journal, trouvera finalement une solution lui permettant de demeurer le seul maître à bord. Cette incursion dans l'histoire de ce journal, rendue possible grâce à une volumineuse correspondance, permet de comprendre un aspect du rôle du réseau ultramontain dans la construction de La Vérité. 


\title{
Articles
}

\section{Un homme et son journal : comment Jules-Paul Tardivel « domestiqua » La Vérité ${ }^{1}$}

\author{
Dominique Marquis \\ Département d'histoire \\ Université du Québec à Montréal
}

\section{Résumé}

En 1881, Jules-Paul Tardivel lance à Québec La Vérité, un journal de combat ultramontain. Tardivel porte ce journal à bout de bras, mais il n'est pas seul : il est soutenu par un réseau ultramontain encore très actif à cette époque. En 1890, Tardivel fait face à des difficultés matérielles telles que la survie du journal est sérieusement mise en péril. Les amis ultramontains de Tardivel sont alors mis à contribution pour trouver une solution. Plusieurs propositions sont évoquées, mais Tardivel, ne voulant pas partager la direction du journal, trouvera finalement une solution lui permettant de demeurer le seul maitre à bord. Cette incursion dans l'histoire de ce journal, rendue possible grâce à une volumineuse correspondance, permet de comprendre un aspect du rôle du réseau ultramontain dans la construction de La Vérité.

\footnotetext{
${ }^{1}$ Cet article fait suite à une communication présentée lors du Congrès annuel de l'Institut d'histoire de l'Amérique française, Sherbrooke, octobre 2012. Je remercie les évaluateurs de la revue Mens pour leurs précieux commentaires.
} 


\section{Abstract}

In 1881, in Quebec City, Jules-Paul Tardivel launches La Vérité, a fiercely ultramontane newspaper. Tardivel is the main operator of this enterprise, but he's not isolated: an ultramontane network that is still fairly active at the end of the 19th century supports him. In 1890, Tardivel is faced with material difficulties that threaten the newspaper's survival. His ultramontane friends are called upon to come up with a solution. Many propositions are on the table, but Tardivel insists on keeping sole control of the newspaper. In the end, a satisfactory arrangement is found that allows La Vérité to continue its crusade under Tardivel's lead. This incursion in the newspaper's history, made possible by an important amount of correspondence, demonstrates the importance of the ultramontane network in the construction of La Vérité as its principal written platform.

Jules-Paul Tardivel est reconnu comme un des plus ardents défenseurs des idées ultramontaines au Québec. Né aux États-Unis en 1851, il termine son cours classique au Séminaire de Saint-Hyacinthe où l'on enseigne abondamment la pensée ultramontaine. Après ses études, il fait un bref retour aux États-Unis, puis il revient au Canada où il acquiert de l'expérience comme journaliste à Saint-Hyacinthe et à Montréal. Installé à Québec à la suite de son mariage, il travaille pour quelques journaux de la ville, dont Le Courrier du Canada et Le Canadien. En juillet 1881, encouragé - moralement et financièrement - par quelques amis, Tardivel publie le premier numéro de $\mathrm{La}$ Vérité, un journal indépendant des partis politiques et entièrement consacré à la défense du catholicisme et de la nation canadiennefrançaise. Pendant plus de vingt ans, soit jusqu’à son décès en 1905 , il a été à la barre de cet hebdomadaire qui tirait à boulets rouges sur tous ceux, clercs ou laïcs, qui s'éloignaient, un tant soit peu, de l'orthodoxie catholique romaine ${ }^{2}$.

2 Pierre Savard, "Jules-Paul Tardivel », dans Dictionnaire biographique du Canada, vol. XIII : 1901-1910, [En ligne], [http://www.biographi.ca/fr/bio/tardivel_jules_ paul_13F.html]. 
L'historiographie québécoise s'est beaucoup intéressée à la question de l'ultramontanisme ${ }^{3}$. Philippe Sylvain a rédigé des études et des synthèses qui ont mis en lumière, entre autres, les premiers lieux de diffusion de la pensée ultramontaine et les principaux antagonismes entre ultramontains et libéraux ${ }^{4}$. Nadia Eid a exploré les liens entre le clergé et le pouvoir politique à travers l'idéologie ultramontaine ${ }^{5}$ et Pierre Rajotte a analysé les stratégies discursives des ultramontains $s^{6}$. Rendant hommage à Philippe Sylvain, plusieurs historiens se sont penchés sur cette question et, dans un ouvrage collectif consacré aux ultramontains canadiens-français, ils ont abordé différentes facettes de cette idéologie qui liait régulièrement les intérêts de l'Église à des enjeux politiques ${ }^{7}$.

Quelques chercheurs ont cependant analysé la question sous un angle différent. Ainsi, René Hardy a utilisé l'exemple des zouaves pontificaux pour illustrer la stratégie du clergé ultramontain, surtout de $\mathrm{M}^{\mathrm{gr}}$ Bourget, dans sa lutte contre les libéraux ${ }^{8}$. Christine Hudon a, quant à elle, analysé le développement du diocèse de SaintHyacinthe pour souligner le processus de transformation, influencé par l'ultramontanisme, de l'action et du discours clérical vers une plus grande uniformisation et un plus grand contrôle des pratiques.

${ }^{3}$ La majorité de ces travaux date de plus de vingt ans. Le sujet de l'ultramontanisme au Québec n'a pourtant pas été épuisé, d'autres approches, notamment celles inspirées de l'histoire culturelle, méritent d'être explorées.

${ }^{4}$ Voir, par exemple, Philippe Sylvain, "Les débuts du "Courrier du Canada" et les progrès de l'ultramontanisme canadien-français ", Cahiers des Dix, vol. 32 (1967), p. 255-278, [En ligne], [http://www.nosracines.ca/f/toc.aspx?id=8777]; et "Quelques aspects de l'antagonisme libéral-ultramontain au Canada français ", dans Jean-Paul Bernard (dir.), Les idéologies québécoises au $19^{\circ}$ siècle, Montréal, Boréal Express, 1973, p. 127-149.

${ }^{5}$ Nadia Fahmy-Eid, Le clergé et le pouvoir politique au Québec : une analyse de l'idéologie ultramontaine au milieu du XIX siècle, Montréal, Hurtubise HMH, 1978.

${ }^{6}$ Pierre Rajotte, Les mots du pouvoir ou le pouvoir des mots : essai d'analyse des stratégies discursives ultramontaines au XIX' siècle, Montréal, L’Hexagone, 1991.

7 Nive Voisine et Jean Hamelin (dir.), Les ultramontains canadiens-français, Montréal, Boréal Express, 1985.

${ }^{8}$ René Hardy, Les zouaves : une stratégie du clergé québécois au XIX siècle, Montréal, Boréal Express, 1980. 
Cette transformation serait à l'origine des démonstrations plus "spectaculaires " de piété qu'on voit apparaître durant la seconde moitié du XIX ${ }^{e}$ siècle 9 . Ces travaux ont su quitter le champ exclusif de l'analyse idéologique pour montrer comment la pensée ultramontaine a fait son chemin dans la culture et dans les pratiques religieuses.

L'idéologie de Jules-Paul Tardivel a suscité l'intérêt de quelques chercheurs. L'étude la plus importante est sans conteste celle de Pierre Savard, publiée en $1967^{10}$. Il y dresse un portrait exhaustif de la pensée du journaliste concernant les relations de la France et des États-Unis avec le Canada français. Grâce à une analyse qui repose en grande partie sur les idées véhiculées par le journal La Vérité, mais qui a aussi fait appel aux archives du journaliste, Savard montre les origines politico-religieuses de la pensée de Tardivel et la constance de son argumentation. Réal Bélanger, quant à lui, s’est intéressé de près au nationalisme de Tardivel ${ }^{11}$. Utilisant surtout le contenu du journal, mais aussi quelques fragments de l'abondante correspondance du journaliste, il soutient que « le nationalisme certes important, [qui] tient en quelque sorte lieu de référent dans la pensée de Jules-Paul Tardivel, ne sert qu'à renforcer la position religieuse et représente en somme l'idéologie d'appoint de son idéologie ultramontaine ${ }^{12}$ ». Il souligne néanmoins l'aspect quelque peu visionnaire de Tardivel dans son projet « indépendantiste », le journaliste ayant ouvert la voie

9 Christine Hudon, Prêtres et fidèles dans le diocèse de Saint-Hyacinthe : 1820-1875, Sillery, Éditions du Septentrion, 1996.

10 Pierre Savard, Jules-Paul Tardivel, la France et les États-Unis (1851-1905), Québec, Les Presses de l'Université Laval, 1967. Pierre Savard a aussi rédigé les notices sur le roman Pour la Patrie, le pamphlet L'Anglicisme, voilà l'ennemi, l'étude Vie du pape Pie IX ses auvres et ses douleurs, les Notes de voyage et les Mélanges ou Recueil d'études religieuses, sociales, politiques et littéraires, parues dans Maurice Lemire (dir.), Dictionnaire des cuvres littéraires du Québec, t. 1 : Des origines à 1900, Montréal, Éditions Fides, 1980, [En ligne], [www.services.banq.qc.ca/sdx/DOLQ/].

11 Réal Bélanger, "Le nationalisme ultramontain : le cas de Jules-Paul Tardivel ", dans Voisine et Hamelin (dir.), Les ultramontains canadiens-français, p. 267-303.

12 Ibid., p. 299. 
à d'autres penseurs du nationalisme, comme Henri Bourassa et Lionel Groulx.

Alors que ces études portaient surtout sur l'idéologie de Tardivel, je m'intéresse davantage à l'histoire du journal La Vérité comme entreprise de presse qu'à son contenu idéologique ${ }^{13}$. Si de nombreux chercheurs ont analysé les journaux sous l'angle de l'idéologie, ultramontaine ou autre ${ }^{14}$, rares sont ceux qui se sont attardés à la structure d'un journal de combat comme La Véritét ${ }^{15}$. Outre les idées bien arrêtées de son directeur sur la vie religieuse et politique, quelle est la nature de l'information et des différents textes que ce journal propose à ses lecteurs? Comment est-il construit ou structuré? Qui accepte de débourser quelques dollars pour acheter de la publicité dans un journal si idéologiquement marqué à une époque où la presse d'information connaît de plus en plus de succès et est certainement un véhicule publicitaire beaucoup plus efficace ${ }^{16}$ ? Un journal de combat témoigne d'une pensée et de valeurs partagées par le rédacteur

13 Voir Dominique Marquis, "Procurer à mes lecteurs quelques heures de délassement" : Les Mélanges, les Notes de voyage et le roman Pour la patrie de Jules-Paul Tardivel ", Journal of the Canadian Historical Association = Revue de la Société historique du Canada, vol. 23, n 1 (2012), p. 157-178; et "Monsieur le rédacteur,..." ou quand les lecteurs de La Vérité prennent la plume : analyse d'une correspondance, 1887-1888 ", dans Guillaume Pinson (dir.), La lettre et la presse : poétique de l'intime et culture médiatique, 2010, [En ligne], [http://www. medias19.org/index.php?id=303].

14 Voir, notamment, Fernand Dumont et al., Les idéologies au Canada français, Québec, Les Presses de l'Université Laval, 1971-1981, 4 t. ; et Philippe Boivin, Le libéralisme du journal Le Soleil, 1896-1911, mémoire de maîtrise (histoire), Québec, Université Laval, 2008.

15 Il faut cependant noter le travail de Christiane Campagna (Le rôle de la presse selon les propriétaires et rédacteurs des journaux montréalais 1830-1880, mémoire de maîtrise (histoire), Montréal, Université du Québec à Montréal, 1998), qui, sans aborder la structure même de la presse d'opinion, se distingue néanmoins des études essentiellement idéologiques par sa volonté de montrer les objectifs multiples que se sont fixés les propriétaires de journaux.

16 Sur le rôle de la publicité dans la presse d'information, voir Jean de Bonville, La presse québécoise de 1884 à 1914 : genèse d’un média de masse, Québec, Les Presses de l'Université Laval, 1988; ou Sébastien Couvrette, Un discours masculin sur la société : la publicité dans les quotidiens québécois des années 1920 aux années 1960, thèse de doctorat (histoire), Montréal, Université du Québec à Montréal, 2009. 
et ses lecteurs; quelles relations ont donc été établies entre Tardivel et les lecteurs de La Vérité? En comparant l'analyse plus approfondie du contenu du journal à celle de la volumineuse correspondance de Tardivel ${ }^{17}$, je compte mettre au jour la nature même du journal ainsi que les réseaux complexes qui l'alimentent ${ }^{18}$. Cette étude de cas permettra de mieux comprendre les moyens pris par les ultramontains de la fin du XIx ${ }^{\mathrm{e}}$ siècle pour maintenir leur espace de discussion dans la cité. Elle mettra aussi en lumière les rouages internes d'un journal de combat publié alors que la transition de la presse d'opinion à la presse d'information est bien amorcée. À la fin du XIx ${ }^{\mathrm{e}}$ siècle, la gestion d'un journal de combat n'est pas toujours chose aisée et l'exemple qui suit souligne la justesse de cette affirmation.

\section{Les souvenirs du directeur}

En 1903, Jules-Paul Tardivel, propriétaire, directeur et principal rédacteur de l'hebdomadaire ultramontain La Vérité depuis 1881, publie le troisième tome de ses Mélanges ou Recueil d'études religieuses, sociales, politiques et littéraires ${ }^{19}$. Il s'agit essentiellement d'une série d'articles qui ont paru dans le journal au cours des années 1883 et 1884, l'objectif étant ici de rappeler à ses fidèles lecteurs que les enjeux liés à la survie de l'Église et de la religion catholiques au Québec sont toujours aussi importants, même vingt ans après les faits $^{20}$. Outre un retour sur la pensée de Tardivel, l'intérêt de ce troisième tome repose aussi sur son introduction d'une cinquantaine de pages dans laquelle il brosse une " histoire sommaire de La Vérité».

17 À ce jour, plus de 1200 lettres, reçues et envoyées par Tardivel, ont été saisies dans une base de données et une analyse exhaustive de leur contenu a été entamée.

18 Une étude semblable a été réalisée autour de l'entreprise du Bien public. Voir Maude Roux-Pratte, Le Bien public, 1909-1978: un journal, une maison d'édition, une imprimerie, Québec, Éditions du Septentrion, 2013. L'auteure souligne comment les réseaux d'Albert Tessier, de Clément Marchand et de Raymond Douville ont été mis à contribution pour assurer le succès et la pérennité de l'entreprise.

19 Jules-Paul Tardivel, Mélanges ou Recueil d'études religieuses, sociales, politiques et littéraires, Québec, Imprimerie S. A. Demers, 1903.

20 Ibid., p. LXVII. 
Tardivel y raconte les grandes lignes de l'histoire du journal ${ }^{21}$, mais, par moments, son texte ressemble aussi à une confession dans laquelle il livre ses états d'âme et ses fréquentes envies de tout laisser tomber, tellement le métier de journaliste catholique est difficile, ingrat et peu lucratif. Sans nommer qui que ce soit, le directeur évoque régulièrement le soutien moral et financier qu'il a reçu de ceux qu'il identifie toujours comme ses amis.

Plus particulièrement, Tardivel témoigne des difficultés financières rencontrées à la fin de l'année 1890 :

En 1890, la Vérité passa par une dernière crise matérielle, la plus forte de toutes, peut-être. À cette époque, l'honorable M. Mercier était au pouvoir, et il était tout-puissant. La Vérité l'avait appuyé en plus d'une circonstance, mais elle était restée absolument indépendante à son égard et blâmait ceux de ses actes qui paraissaient dignes de blâme. Cette indépendance déplut-elle à M. Mercier lui-même et résolut-il de créer des embarras à la Vérité? Je ne saurais l'affirmer. Toujours est-il que certains intrigants, qui tenaient de près au cabinet, réussirent à persuader MM. L. Drouin \& Frère que leur commerce de librairie irait mieux, qu' ils recevraient de fortes commandes du gouvernement s'ils cessaient d'administrer la Vérité et même de lui donner l'hospitalitée ${ }^{22}$.

Il importe de préciser ici que, depuis novembre 1881, le journal est administré par la firme L. Drouin \& Frère, libraires et imprimeurs ayant pignon sur la rue Saint-Joseph dans le quartier Saint-Roch. Cette entreprise veille à imprimer et à expédier le journal et elle gère la vente d'annonces et la collecte des abonnements. Tardivel y loue un petit bureau. Il n'a donc pas à se soucier des tracas administratifs, et il consacre tout son temps à la rédaction du journal et à la correction des épreuves ${ }^{23}$. La menace, qui aurait émané de cercles entourant le premier ministre Mercier, a été prise au sérieux et le lien d'affaires qui unissait Tardivel et les frères Drouin depuis neuf ans a été rompu.

\footnotetext{
21 Ibid., p. V-LIX.

22 Ibid., p. IX-X.

23 Ibid., p. VII.
} 
Non seulement Tardivel doit désormais s'occuper seul de l'administration du journal, mais il doit aussi chercher une imprimerie et un autre local pour loger La Vérité. Étant, de son propre aveu, un piètre administrateur, il raconte la suite de l'aventure :

[...] je commençai par un faux pas. Dans l'espoir d'améliorer la situation du journal, [...] je voulus agrandir le champ de mes opérations en créant une imprimerie d'ouvrages de ville. Grâce à la générosité de quelques amis, à qui j'avais fait part de mon projet, je montai, à Saint-Roch, à des frais assez considérables, un atelier d'imprimerie générale.

Me voilà donc directeur, rédacteur en chef et administrateur du journal et, par surcroit, chef d'imprimerie.

C'était une folle entreprise. Heureusement saint Joseph qui a toujours été le véritable homme d'affaires de la Vérité, me fit comprendre, à temps, qu'à vouloir conduire deux besognes à la fois je les gâcherais inévitablement toutes deux. Il m’inspira l'idée de me faire petit, au lieu de vouloir me faire grand. J'abandonnai donc, le plus tôt possible, mais non sans d'assez lourdes pertes, ma téméraire entreprise d'agrandissement qui eût fini par m'entraîner à la ruine complète : et je résolus de m’occuper à l'avenir exclusivement du journal. Je transportai bureaux et ateliers à la maison que, grâce à la générosité d'autres amis, je venais d'acquérir sur les hauteurs de Ste-Foye, près de la ville. J'installai la Vérité dans la cave et en fit une ouvre domestique, très modeste, mais solide ${ }^{24}$.

Si le récit de cette aventure publié dans le bref historique du journal est plutôt laconique et se résume à quelques paragraphes, la correspondance entre Tardivel et ceux qu'il nomme ses amis montrent que la situation fut plus complexe et que les enjeux étaient, aux yeux de Tardivel, liés autant à la direction du journal qu'à sa survie. Comment sauver un journal de combat du naufrage? Un tel journal

${ }^{24}$ Ibid., p. X-XI. 
peut-il être dirigé par plusieurs têtes? L'article qui suit présente cet épisode de l'histoire administrative de La Vérité.

\section{Des débuts difficiles}

Rappelons que Jules-Paul Tardivel fonde La Vérité en juillet 1881 non seulement pour dire toute la vérité, mais aussi pour combattre l'erreur, peu importe sa provenance ${ }^{25}$. Dans son programme, il affirme qu'il n'adhérera à aucun parti afin de maintenir sa liberté et son impartialité. Il ne s'attaquera pas aux hommes, mais aux idées. Il ne prétend toutefois pas usurper la place des évêques, puisqu'il ne souhaite pas s'élever au-dessus du rang que sa position de laïc lui impose. Il affirme que plusieurs écrivains " de talents " ont déjà signifié leur intérêt pour son journal et qu'ils feront partie des collaborateurs réguliers, mais lui seul en assurera l'entière direction ${ }^{26}$.

Très au fait des difficultés financières vécues par de nombreux journaux parce que plusieurs lecteurs négligent le paiement de leur abonnement, Tardivel aborde ainsi cette épineuse question :

Comme nous voulons que notre journal vive et prospère, nous avons pris la ferme résolution d'exiger que tous les abonnements soient payés d'avance. [...] Que ceux qui ne nous croient pas, [sic] essaient d'obtenir le journal sans payer l'abonnement d'avance! Le système que suivent les journaux en ce pays est ruineux. Pour notre part, nous sommes décidés à en finir avec la routine. Nous aimons mieux dix abonnés qui paient d'avance, que cinquante qui ne paient que lorsqu'on les tire par l'oreille. [...] tout homme qui a le moyen de payer la somme de $\$ 1.50$ [alors le coût d'un abonnement d'un an] dans le cours de l'année, peut trouver ce montant à un moment donné. Ainsi, encore une fois, payable d'avance sans aucune exception ${ }^{27}$.

${ }^{25}$ L'expression « combattre l'erreur " a été fréquemment utilisée pour désigner la dénonciation de tout ce qui dérogeait à l'orthodoxie catholique.

${ }^{26}$ La Vérité, 14 juillet 1881, p. 1.

${ }^{27}$ Ibid. 
Malheureusement pour Tardivel, il a beau vouloir « en finir avec la routine ", il doit rapidement faire face au même problème que la plupart de ses concurrents : les abonnés négligent de payer leur dû et les soucis financiers commencent à hanter le directeur du journal. Tardivel, marié et père de cinq enfants, réussit difficilement à vivre décemment de cette unique plume. Il se plaint d'ailleurs régulièrement de cette situation pour le moins inconfortable. Afin de combler le manque à gagner, il accepte des contrats de traduction ${ }^{28}$ - il traduit notamment le roman de Robert Louis Stevenson The Strange Case of Dr Jekyll and $\mathrm{Mr} \mathrm{Hyde^{29 }}$ - et il devient même, durant quelques années, chroniqueur parlementaire à la pige pour le journal L'Étendard de Montréal.

Tardivel et La Vérité bénéficient aussi du soutien des " amis". L'abondante correspondance du directeur témoigne de fréquents épisodes de découragement durant lesquels il songe sérieusement à tout abandonner. Par exemple, en novembre 1889, Tardivel se plaint à son ami Georges-Allan Bourgeois de l'incompréhension dont fait preuve son mentor, le père Joseph Grenier :

Le bon Père n'ayant jamais eu les soucis d'élever une famille ne peut pas se rendre compte de la situation d'un père de cinq enfants qu'il faut nourrir, vêtir et instruire à même un journal qui n'a jamais donné à son propriétaire et rédacteur en chef, après huit années d'un travail opiniâtre, plus de \$ 15 par semaine, [...]. Il me dit que je suis tenté par le démon du découragement. Je veux bien le croire; mais enfin, il me semble que mon premier devoir est de chercher un moyen de gagner la vie de ma famille ${ }^{30}$.

Invariablement, chaque fois qu'il exprime de tels sentiments, les amis lui rappellent l'importance de poursuivre sa mission en dépit

28 Journal de Jules-Paul Tardivel, vol. 2 (1884-1886), Bibliothèque et Archives nationales du Québec, Centre d'archives de Québec (ci-après BAnQ-Q), Fonds Jules-Paul Tardivel, P683.

29 Savard, "Jules-Paul Tardivel ».

30 Lettre de Jules-Paul Tardivel à Georges-Allan Bourgeois, 29 novembre 1889, BAnQ-Q, Fonds Jules-Paul Tardivel, MSS225. 
des sacrifices exigés et ils l'assurent de leur soutien ${ }^{31}$. Outre ces paroles d'encouragement, ce soutien se traduit généralement par d'importants dons en argent. Le journal n'étant pas rentable, à maintes reprises des campagnes de souscription sont lancées pour en assurer la survie. Mais les amis, parmi lesquels on compte plusieurs médecins et avocats résidant autant à Montréal et à Trois-Rivières qu’à Québec ${ }^{32}$, vont plus loin que de simplement maintenir le journal à flot : en 1888, ils offrent au journaliste un voyage de sept mois en Europe, afin qu'il puisse rencontrer les plus grands penseurs catholiques ${ }^{33}$, voyage dont il fera profiter ses lecteurs en faisant parvenir chaque semaine au journal ses impressions de voyage ${ }^{34}$. De plus, en 1890, alors que les frères Drouin annoncent qu'ils doivent laisser tomber La Vérité, une autre campagne de souscription est déjà en cours, campagne dont l'objectif est, cette fois-ci, de permettre à Tardivel d'acheter la maison du chemin Sainte-Foy qu'il occupe avec sa famille.

\section{À la recherche d'une solution}

En juillet 1890, la nouvelle du désengagement de Drouin \& Frère à l'endroit de La Vérité a donc l'effet d'une petite bombe. Il faut rapidement trouver une solution. Le processus de consultation est enclenché. La première réaction de Tardivel est plutôt sereine : dans une lettre au D $\mathrm{D}^{\mathrm{r}}$ Louis-Édouard Desjardins ${ }^{35}$, un ami de Montréal,

31 Lettre de Georges-Allan Bourgeois à Jules-Paul Tardivel, 2 janvier 1888, Archives des Jésuites au Canada (AJC), Fonds Jules-Paul Tardivel, BO-170-2, 1.

32 Les noms rencontrés le plus fréquemment dans la correspondance de Tardivel sont Georges-Allan Bourgeois de Trois-Rivières, Louis-Édouard Desjardins, Philippe Landry et Gustave Lamothe de Montréal, les D ${ }^{\text {rs }}$ Boulet, Garneau et Sanson de Québec, sans oublier le père Joseph Grenier, s.j.

33 "Départ pour l'Europe ", La Vérité, 8 septembre 1888, p. 58.

${ }^{34}$ Ces notes seront publiées chaque semaine dans le journal et elles seront reprises dans un ouvrage, Notes de voyage en France, Italie, Espagne, Irlande, Angleterre, Belgique et Hollande, Montréal, Eusèbe Sénécal \& fils, 1890.

35 Louis-Édouard Desjardins (1837-1919). Médecin, il est un des pionniers de l'ophtalmologie au Québec. Il prendra position en faveur de l'École de médecine et de chirurgie de Montréal contre les volontés assimilatrices de l'Université Laval. Les rencontres et les échanges de lettres sont fréquents entre les deux hommes. 
il estime que la situation n'est pas critique et que de ce contretemps pourrait émerger quelque chose de bien : « je suis convaincu que c'est un désagrément momentané pour un bien considérable. " "Il serait assez facile de profiter de cette circonstance imprévue pour mettre La Vérité sur un pied plus solide et plus satisfaisant. " Il suggère alors de créer une société par actions qui deviendrait propriétaire du journal et qui pourrait embaucher un gestionnaire pour occuper la fonction de gérant administratif. Il prend l'exemple de L'Univers, journal ultramontain parisien fondé par Louis Veuillot, qui fonctionne de cette manière et qui s'en tire très bien. Évidemment, pour Tardivel, il n'est pas question de laisser cette propriété entre les mains de n’importe qui. Les actions de la société ne seraient pas offertes à des "gens quelconques", mais à des amis, à des gens sûrs. Aucun homme politique, qu'il soit bleu ou rouge, ne serait admis dans la société : le journal doit demeurer indépendant, et ses propriétaires doivent l'être aussi. Il est conscient que les profits ne seraient pas immédiatement au rendez-vous, mais il estime que le projet est réaliste ${ }^{36}$.

Tardivel soumet aussi, en même temps, cette solution au père Joseph Grenier, un jésuite qui est à la fois son mentor, son confident et son conseiller, mais qui se révèle aussi ce « saint Joseph » qui aurait toujours été "le véritable homme d'affaires de La Vérité", comme le mentionne Tardivel dans son histoire du journal. Dans une lettre adressée au jésuite, qui est alors en mission au Sault-Sainte-Marie, il présente son projet de société par actions et insiste sur le fait qu'il s'agit de la seule solution qui lui permette de se consacrer uniquement à la rédaction du journal en le libérant du poids de l'administration et que, de surcroît, cette solution pourrait devenir une occasion de développement. "Il serait possible, dit-il, avec une administration soignée et bien surveillée, et en augmentant un peu le matériel du journal afin de pouvoir faire quelques impressions, de trouver dans

36 Lettre de Jules-Paul Tardivel à Louis-Édouard Desjardins, 23 juillet 1890, BAnQ-Q, Fonds Jules-Paul Tardivel, MSS225. 
le journal de quoi payer les dépenses et de donner au rédacteur un salaire convenable ${ }^{37}$."

Gustave Lamothe, avocat montréalais et administrateur du journal L'Étendard, est aussi consulté. Tardivel le connait de longue date et il a confiance en son jugement, notamment parce qu'il est en voie de tirer le quotidien ultramontain d'une situation difficile à la suite du décès du sénateur François-Xavier-Anselme Trudel, fondateur et propriétaire de L'Etendard ${ }^{38}$. Tardivel expose donc son projet à l'avocat et il lui demande de le conseiller sur les procédures à suivre, en lui fournissant quelques chiffres sur la situation financière de $L a$ Vérité. Le journal n'est pas très endetté et surtout, si les 1500 abonnés payent leur dû (un abonnement coûte alors deux dollars par année) et qu'on ajoute les revenus d'annonces et de travaux d'impression de $500 \$$, il serait possible, alors que les dépenses (papier, typographes et administration) s'élèvent à environ 2150 \$, de dégager un surplus de 1350 \$ par année, ce qui serait nettement suffisant pour payer un loyer acceptable et un salaire décent au rédacteur ${ }^{39}$.

Selon Tardivel, cette solution nécessiterait toutefois des investissements importants dans l'imprimerie et une surveillance beaucoup plus étroite de l'administration, en assurant plus particulièrement un meilleur suivi du paiement des abonnements. L'option de la société par actions est la seule possible puisqu' elle offrirait aux amis de Tardivel qui l'ont fréquemment aidé à assurer la survie du journal une participation à d'éventuels profits. Comme Tardivel ne veut plus quémander, cette solution lui garantirait une certaine autonomie financière :

Je cherche surtout, en favorisant cette compagnie, à être déchargé de toute responsabilité vis-à-vis des amis qui m’ont aidé par le

${ }^{37}$ Lettre de Jules-Paul Tardivel au père Joseph Grenier, 24 juillet 1890, BAnQ-Q, Fonds Jules-Paul Tardivel, MSS225.

38 Tardivel ayant été sollicité pour prendre la relève de Trudel à Montréal et ayant décliné l'invitation, Lamothe a en effet réussi à réorganiser le journal en collaborant étroitement avec le fils du sénateur.

39 Lettre de Jules-Paul Tardivel à Gustave Lamothe, 25 juillet 1890, BAnQ-Q, Fonds Jules-Paul Tardivel, MSS225. 
passé à maintenir le journal. Je crois avoir fait un bon usage de l'aide reçue, mais la situation sera plus satisfaisante pour tout le monde, lorsque ceux qui soutiennent le journal auront leur part légitime dans sa direction ${ }^{40}$.

Tardivel consulte ainsi plusieurs amis; il cherche des conseils et des appuis. Il suggère au père Grenier de prendre une partie de l'argent destiné à l'achat de sa maison de Sainte-Foy pour relancer le journal. Toujours animé d'un esprit de sacrifice, Tardivel poursuit en affirmant qu'à ses yeux, l'achat de cette maison, qui ne profiterait qu'à lui et à sa famille, est moins urgent que la survie du journal ${ }^{41}$. Dans une lettre au $\mathrm{D}^{\mathrm{r}}$ Louis-Édouard Desjardins, il précise que Grenier n'est pas d'accord avec cette proposition. Le jésuite aurait demandé à Tardivel de ne pas s'engager prématurément dans le processus de création d'une société par actions. Il faudrait, selon lui, attendre que la souscription lancée en faveur de l'achat de sa maison soit terminée ${ }^{42}$. Tardivel sonde le terrain auprès de Desjardins : que doit-il favoriser, le journal ou lui-même? Il ose croire qu'il serait possible de mener les deux campagnes de front.

Toutefois, seules deux options lui paraissent plausibles : donner un nouvel élan au journal en créant cette société qui lui permettrait de grossir les installations et de diversifier l'offre de services afin de générer plus de revenus, ou en suspendre définitivement la publication. Ce n'est pas de gaieté de cœur qu'il en arrive à ce constat. Ne souhaitant pas vraiment mettre fin aux activités du journal, et contre l'avis du père Grenier, Tardivel lance donc le processus de création d'une société d'actionnaires. Le 9 septembre, il écrit au $\mathrm{D}^{\mathrm{r}}$ Desjardins :

Si nous voulons que La Vérité se maintienne, il faut absolument agrandir un peu le cadre de ses opérations, établir un atelier

40 Ibid.

${ }^{41}$ Lettre de Jules-Paul Tardivel au père Joseph Grenier, 30 juillet 1890, BAnQ-Q, Fonds Jules-Paul Tardivel, MSS225.

${ }^{42}$ Lettre de Jules-Paul Tardivel à Louis-Édouard Desjardins, 20 août 1890, BAnQ-Q, Fonds Jules-Paul Tardivel, MSS225. 
d'impressions qui aide le journal à vivre. Or cet atelier ne peut s'établir sans un capital additionnel de 1500 \$ à 2000 \$ et je ne l'ai pas. Si je l'avais, je l'y risquerais, car dans les affaires de journalisme, une seule tête vaut mieux que plusieurs, c'est incontestable ${ }^{43}$.

Il aimerait demeurer le seul maître à bord et ne pas avoir à quémander encore une fois des fonds supplémentaires, mais la situation très précaire dans laquelle il se trouve l'oblige à envisager ce nouvel arrangement.

Les échanges de lettres sont nombreux : plusieurs correspondants émettent des réserves à propos du projet de société d'actionnaires. Tardivel lance donc cette menace : "Si l'on ne peut pas mettre la Vérité sur un meilleur pied, je vais la laisser tomber. "Cette menace est répétée plusieurs fois : si aucune solution n'est trouvée, le journal cessera de paraître le 31 octobre, date d'expiration du contrat des typographes et de l'entente avec les frères Drouin ${ }^{44}$. Tardivel demande même à son grand ami, le $\mathrm{D}^{\mathrm{r}}$ Georges-Allan Bourgeois, inspecteur des postes à Trois-Rivières, de l'aider à se trouver un nouvel emploi. Bourgeois s'empresse de lui offrir son " concours " même s'il estime que, Tardivel n'étant certainement pas prêt à accepter un emploi pour l'un ou l'autre gouvernement, la démarche ne sera pas facile ${ }^{45}$.

Pourtant, malgré toutes ces incertitudes, Tardivel va de l'avant. En effet, dès le 13 septembre, une offre d'emploi pour un apprenti typographe paraît dans le journal ${ }^{46}$ et à la fin de septembre, La Vérité annonce que ses nouveaux ateliers pourront très bientôt réaliser "toutes sortes d'impressions " ${ }^{47}$. Durant tout le mois d'octobre, les lecteurs sont avisés que les bureaux du journal déménageront bientôt

43 Lettre de Jules-Paul Tardivel à Louis-Édouard Desjardins, 9 septembre 1890, BAnQ-Q, Fonds Jules-Paul Tardivel, MSS225.

${ }^{44}$ Lettre de Jules-Paul Tardivel au père Joseph Grenier, 25 septembre 1890, BAnQ-Q, Fonds Jules-Paul Tardivel, MSS225.

45 Lettre de Georges-Allan Bourgeois à Jules-Paul Tardivel, $1^{\text {er }}$ octobre 1890, AJC, Fonds Jules-Paul Tardivel, BO-170-2, 1.

46 La Vérité, 13 septembre 1890, p. 62.

47 La Vérité, 27 septembre 1890, p. 80. 
et qu'on y offrira désormais des services d'imprimerie générale. On demande aux lecteurs de confier des mandats et de réserver l'impression de leurs ouvrages pour la nouvelle imprimerie, mais surtout, en ces temps difficiles, on leur demande de payer leur abonnement. Cette annonce sera publiée toutes les semaines et on insistera beaucoup sur le besoin pressant d'argent.

Ainsi, pendant que dans les pages de La Vérité on affiche un certain optimisme en annonçant le déménagement prochain du journal et de nouvelles activités commerciales, une part de pessimisme anime les échanges entre Tardivel et ses proches. Même s'il poursuit ses démarches en vue d'acquérir l'équipement nécessaire et sollicite ses connaissances pour obtenir des contrats d'impression - il communique notamment avec le curé David Gosselin, responsable de la publication de La Semaine religieuse de Québec pour lui demander de confier l'impression de l'hebdomadaire à ses ateliers ${ }^{48}$-, le financement de la nouvelle société ne progresse pas très rapidement. Pire encore, Tardivel perçoit une certaine indifférence de la part des abonnés. En effet, plutôt que d'encourager les abonnés à régler leurs dettes envers le journal, les rappels lancés dans le journal incitent plusieurs d'entre eux à mettre fin à leur abonnement. Le 8 octobre, Tardivel se confie au père Grenier :

N'y a-t-il pas lieu d'examiner sérieusement s'il n'y a pas, dans cette indifférence si générale, si persistante, qui augmente toujours, au lieu de diminuer, un signe, que pour une raison ou pour une autre, le bon Dieu ne veut pas que l'œuvre continue. Je n'ai pas le moindre doute sur l'excellence de la cause que la Vérité défend; mais il peut entrer dans les desseins de la Providence que cette cause ne soit pas défendue ou ne le soit pas par moi ${ }^{49}$.

48 Lettre de Jules-Paul Tardivel au curé David Gosselin, 15 octobre 1890, BAnQ-Q, Fonds Jules-Paul Tardivel, MSS225.

${ }^{49}$ Lettre de Jules-Paul Tardivel au père Joseph Grenier, 8 octobre 1890, BAnQ-Q, Fonds Jules-Paul Tardivel, MSS225. 
Les desseins de la Providence n’ont peut-être pas été exprimés assez clairement puisque Tardivel poursuit ses démarches et, le 12 octobre, il annonce au père Grenier et au $\mathrm{D}^{\mathrm{r}}$ Desjardins qu'il a trouvé un local et qu'il prépare son déménagement pour le début de novembre. Il précise qu'il a toujours besoin de soutien et il espère que ses amis trouveront les moyens pour l'aider à redresser le journal et sa propre situation financière. En effet, Tardivel aura besoin de 2500 \$ supplémentaires pour faire fonctionner le journal et nourrir sa famille. Des sommes d'argent lui ont déjà été remises, mais elles sont nettement insuffisantes pour couvrir les frais engagés pour l'achat d'une presse et des caractères nécessaires aux différents travaux de ville ${ }^{50}$.

Malgré les multiples précautions qu'il compte prendre pour "faire un choix éclairé » des actionnaires initiaux, Tardivel s'inquiète toujours du risque que les actions d'une éventuelle société puissent tomber entre des mains indésirables, voire ennemies, que ce soit par leur vente ou simplement par leur cession. Il semble difficile d'avoir l'assurance qu'une telle éventualité ne survienne pas. Devant l'hésitation de ses proches à s'engager dans le projet de société par actions, Tardivel lance une perche en proposant une nouvelle solution, mitoyenne : entre la souscription à « fonds perdus » qui lui permettrait de demeurer seul maître à bord et la société d'actionnaires face auxquels il lui faudrait rendre régulièrement des comptes et même se plier à certaines exigences, il serait possible de créer une sorte de société d'amis. Il conserverait le contrôle absolu et exclusif du journal, mais il s'engagerait à soumettre annuellement à ceux qui auraient fourni le capital nécessaire à la réorganisation de La Vérité, un état détaillé des affaires du journal. Si, après s'être payé un salaire raisonnable estimé à 1200 \$ par année, il restait des profits, ils seraient distribués sous forme de bonus aux contributeurs. De plus, si les affaires du journal reprenaient un réel élan, Tardivel pourrait rembourser entièrement la mise de fonds des investisseurs, se libérant ainsi de

${ }^{50}$ Lettres de Jules-Paul Tardivel au père Joseph Grenier et à Louis-Édouard Desjardins, 12 octobre 1890, BAnQ-Q, Fonds Jules-Paul Tardivel, MSS225. 
toute obligation envers eux ${ }^{51}$. Cette proposition montre à quel point le directeur de La Vérité cherche désespérément une solution, mais aussi comme il est frileux à l'idée de devoir partager le contrôle du journal avec d'autres, même s'il s'agit d'amis sûrs.

Tardivel tentera même sa chance auprès d'Eusèbe Sénécal, l'imprimeur montréalais avec lequel il a fait affaire pour la publication de ses Notes de voyage. Il lui propose de prendre en charge l'administration et l'impression du journal, un peu comme le faisaient les frères Drouin. Sa lettre détaille les conditions d'un éventuel contrat de plusieurs années $^{52}$. La réponse de Sénécal n'a pas été conservée dans les archives, mais manifestement cette demande n'a pas été reçue favorablement, et aucun des deux hommes ne semble y avoir donné suite.

La proposition, entre "l'action et le don ", ne recevant pas l'assentiment des principaux intéressés, et même si une solution définitive n'est pas encore trouvée, Tardivel poursuit l'organisation de la nouvelle imprimerie. Le journal annonce finalement son déménagement à la fin de novembre. On précise alors que les installations sont prêtes et que l'imprimerie possède un bel assortiment de caractères "très complet et dans les derniers goûts " et qu'on peut rapidement livrer aux curés les cahiers nécessaires au recensement de leur paroisse à bon prix. On rappelle toujours l'importance de payer son abonnement ${ }^{53}$.

Confiné au Sault-Sainte-Marie où il est en mission, le père Grenier suit de loin les mésaventures de son protégé. Rappelons qu’il n'approuve pas le projet de société par actions, et il le met en garde : plusieurs souscripteurs ont signifié leur refus de modifier les objectifs de leur contribution, ils pourraient retirer leur don si cet argent n'était

51 Lettre de Jules-Paul Tardivel à Louis-Édouard Desjardins, 17 octobre 1890, BAnQ-Q, Fonds Jules-Paul Tardivel, MSS225; Lettre de Jules-Paul Tardivel au père Joseph Grenier, 18 octobre 1890.

52 Lettre de Jules-Paul Tardivel à Eusèbe Sénécal, 28 décembre 1890, BAnQ-Q, Fonds Jules-Paul Tardivel, MSS225.

53 La Vérité, 29 novembre 1890, p. 150-151. 
pas destiné à l'achat de la maison ${ }^{54}$. Les affaires s'annoncent très mauvaises pour La Vérité et la nouvelle imprimerie. Tardivel avoue avoir agi avec imprudence en investissant trop rapidement dans un grand atelier d'imprimerie, et il est découragé. Les appuis se font plus rares, il craint que ses amis ne l'abandonnent. Il a des difficultés à faire face à ses obligations et il n'arrive toujours pas à se payer un salaire. Il avoue au père Grenier avoir commis une erreur en ne suspendant pas la publication du journal; il est conscient de ses piètres qualités de gestionnaire, mais il demeure convaincu de la validité de sa proposition de créer une société d'actionnaires :

Décidément je ne suis pas fait pour être à la tête d'une entreprise comme celle que j'ai sur les bras. Je ne suis pas homme d'affaires le moins du monde. À [vouloir] conduire les affaires matérielles du journal et de l'imprimerie, je vais ruiner ma santé et échouer misérablement, j'en ai peur. Si on avait pu ou voulu accepter mon projet de former une société qui m’eut déchargé de toute responsabilité financière, c'eût été infiniment mieux ${ }^{55}$.

\section{La domestication du journal}

Jusqu’à la fin de l'année 1890, quelques amis et lecteurs fidèles à la cause s'affairent à ramasser des fonds, mais les sommes sont insuffisantes ${ }^{56}$. La concurrence étant vive sur le marché québécois des travaux de ville, les commandes d'impression ne sont pas au rendezvous. Le journal souffre énormément de la situation parce que, trop occupé par la gestion de l'imprimerie, le rédacteur ne peut se consacrer entièrement aux choses essentielles à son métier : il ne peut se tenir au courant $\mathrm{du}$ " mouvement des esprits dans le monde

54 Lettre de Jules-Paul Tardivel à Georges-Allan Bourgeois, 23 novembre 1890, BAnQ-Q, Fonds Jules-Paul Tardivel, MSS225.

55 Lettre de Jules-Paul Tardivel au père Joseph Grenier, 6 décembre 1890, BAnQ-Q, Fonds Jules-Paul Tardivel, MSS225.

56 La correspondance de Tardivel compte plusieurs dizaines de lettres dans lesquelles le journaliste remercie des lecteurs de leur contribution pécuniaire. Les sommes reçues oscillent entre 5 \& et $100 \$$. 
civilisé, des événements des deux hémisphères ". La situation est presque catastrophique. Le directeur de La Véritésent même le besoin de s'excuser auprès de ses lecteurs :

Nous comptions bien rencontrer des difficultés dans nos travaux de déménagement et d'installation; car l'expérience était là pour nous avertir de ne point nous attendre au succès, même relatif. [...] Mais, franchement, depuis quelques semaines les déceptions et les obstacles se multiplient au-delà de nos prévisions. [...] Nous osons donc espérer que nos lecteurs voudront bien se montrer indulgents. De notre côté, nous ferons toute la diligence possible et n'épargnerons aucun effort pour mettre notre journal en état de poursuivre sa mission ${ }^{57}$.

Puis, soudainement, Tardivel envisage l'avenir de La Vérité autrement. Il écrit au père Grenier en janvier 1891 : «Le bon Dieu, voyant, je suppose, que la leçon était suffisante, m’envoie une de ces idées nettes et claires qu'illuminent [sic] l'esprit subitement. Au lieu de vouloir faire grand dans l'espoir d'augmenter les recettes du journal, je vais faire petit afin d'en réduire les dépenses. " Il veut désormais installer son atelier dans sa maison du chemin Sainte-Foy. Il pourra ainsi réduire son personnel au minimum en ne conservant qu'un compagnon typographe et un apprenti. De plus, ayant reçu l'approbation de son épouse, il compte impliquer ses filles dans les affaires courantes du journal en leur demandant de s'occuper de la préparation des exemplaires, des envois postaux et de la gestion des listes d'abonnés. De cette manière, il estime que les dépenses n'excéderont pas 20 \$ par semaine, soit environ 1000 \$ par année. Avec la possibilité, plus réaliste, de conserver 1000 abonnés à deux dollars par année, il réussira ainsi à faire vivre le journal et sa famille ${ }^{58}$. Il confesse au père Grenier sa sottise et son erreur et il fait preuve d'humilité en avouant que

57 "À nos lecteurs ", La Vérité, 10 janvier 1891, p. 1.

58 Il est à noter que dans l'historique publié dans le troisième tome des Mélanges, Tardivel laisse entendre que l'idée lui aurait été inspirée par « saint Joseph ", alors que la correspondance montre bien que Tardivel a trouvé lui-même la solution et la présente au père Grenier. 
« [p]our réaliser ce projet avec avantage, il faut que j'achète la propriété où je suis car je ne veux pas être dérangé au bout d'un an. C'est ainsi que, après vous avoir fait des reproches parce que vous ne vouliez pas lâcher votre plan, nous y sommes forcément ramenés ${ }^{59}$ ". Le 17 janvier 1891, La Vérité publie une annonce : une presse à cylindre et un couteau " tout neuf " pour couper le papier sont à vendre.

Le projet de société par actions est donc définitivement abandonné - il était de toute manière voué à l'échec - et Tardivel devient, grâce à la campagne de souscription lancée en sa faveur, propriétaire de la maison du chemin Sainte-Foy. Le 7 février 1891, la une de $L a$ Vérité annonce la nouvelle installation. Avec une franchise étonnante, Tardivel explique à ses lecteurs que la nouvelle imprimerie générale ne lui causait que des embarras et que les économies réalisées lui permettront de « joindre les deux bouts » et de s' « occuper sérieusement de la rédaction du journal ». Tout devrait rentrer dans l'ordre dans un mois et les « lecteurs n'auront qu'à se féliciter du changement ${ }^{60}$ ". Une telle manière d'exposer une situation difficile souligne le caractère plus «familial ", voire plus intime de ce journal de combat. Tardivel ne s'adresse pas à une masse anonyme, mais comme au sein d'une grande famille unie, il partage ses craintes, ses erreurs et ses espoirs avec ses lecteurs.

\section{Conclusion}

Cet épisode de l'histoire de La Vérité est éclairant à plusieurs égards : d'une part, il constitue un bon exemple de l'activisme du réseau ultramontain quand survient un événement pouvant nuire à la cause $^{61}$. Dès que Tardivel annonce que les frères Drouin mettront fin à leur entente, les ultramontains, qui comptent sur lui pour être leur

59 Lettre de Jules-Paul Tardivel au père Joseph Grenier, 16 janvier 1891, BAnQ-Q, Fonds Jules-Paul Tardivel, MSS225.

${ }^{60}$ La Vérité, 7 février 1891, p. 1.

${ }^{61}$ La recherche que j'effectue actuellement sur Tardivel et le journal La Vérité permettra de définir avec plus de précision les contours exacts de ce réseau. 
porte-parole, s'animent et cherchent une manière de permettre à La Vérité de poursuivre sa mission. Ils ne sont pas tous d'accord sur la solution à apporter, mais tous s'entendent pour dire qu'il ne faut pas que La Vérité disparaisse : elle est leur voix. Certains délient considérablement les cordons de leur bourse, notamment le $\mathrm{D}^{\mathrm{r}}$ Louis-Édouard Desjardins qui versera plusieurs centaines de dollars pour soutenir le journal, d'autres évoquent même la possibilité de déménager Tardivel, sa famille et son journal à Montréal si cela peut en assurer la survie.

D'autre part, cet épisode est aussi significatif pour comprendre le rôle du directeur dans une entreprise de presse comme un journal de combat. Tardivel est le pilier central de ce journal. Même s'il a besoin d'appuis, financiers et moraux, il est très réticent à partager la direction de l'hebdomadaire. À maintes reprises, il affirme que $L a$ Vérité ne peut pas être dirigée par plusieurs têtes. Bien que la société par actions apparaisse comme une solution raisonnable, Tardivel lui-même la remet fréquemment en question en évoquant le risque d'une perte de pouvoir. Malgré ses fréquentes périodes de découragement, alors qu'il pense à tout abandonner et qu'il aurait peut-être besoin d'aide, il tient mordicus à assumer seul la direction et pratiquement toute la rédaction du journal. Ce journal est son journal, et après six mois de tergiversations, sa domestication ${ }^{62}$ lui permet d'en demeurer le seul maître.

Abnégation ou entêtement? Peut-être un peu des deux, mais Tardivel croit à la cause qu'il défend tout autant qu'il tient à son indépendance. Il compte néanmoins sur son réseau pour le soutenir au besoin et le nourrir en informations diverses. Ainsi, afin d'enrichir sa réflexion, il discute abondamment des sujets jugés délicats avec ses correspondants, et ces discussions sont, par la suite, transposées

62 Tardivel lui-même utilise le terme " domestication " pour indiquer son choix de transférer les activités du journal - administration, rédaction et impression - dans son domicile familial. 
dans le journal ${ }^{63}$. L'analyse croisée de la correspondance et du journal révélera ce réseau complexe de relations déjà évoqué par Pierre Savard ${ }^{64}$, mais jamais dessiné avec précision. Une chose est certaine, à la suite de cet épisode, la question de l'administration du journal ne se posera plus jamais de manière aussi aiguë et Tardivel, en ayant réduit l'ampleur de ses activités, pourra maintenir son œuvre à flot. Il demeure le seul maître à bord jusqu’à son décès en 1905, en dépit des épisodes plus difficiles qui continueront à jalonner son parcours professionnel.

63 Une première analyse de cette correspondance a paru dans Marquis, «"Monsieur le rédacteur,..." ou quand les lecteurs de La Vérité prennent la plume ».

64 Pierre Savard affirme, dans la notice du Dictionnaire biographique du Canada, que "Tardivel n'a rien d'un isolé qui n'écoute que lui-même » et qu'il a su bien s'entourer (Savard, " Jules-Paul Tardivel »). 